\title{
Human therapeutic cloning, pitfalls and lack luster because of rapid developments in induced pluripotent stem cell technology
}

\author{
Song Hua ${ }^{\mathrm{a}, \mathrm{b}}$, Henry Chung ${ }^{\mathrm{a}}$, Kuldip Sidhu ${ }^{\mathrm{a}}$ \\ aStem Cell Lab, Faculty of Medicine, School of Psychiatry, University of New South Wales, New \\ South Wales 2052, Australia, ${ }^{b}$ College of Veterinary Medicine, Northwest A\&F University, Yangling, \\ Shaanxi 712100, China
}

\begin{abstract}
Background: Therapeutic cloning is the combination of somatic cell nuclear transfer (SCNT) and embryonic stem cell (ES) techniques to create specific ES cells that match those of a patient. Because ES cells derived by nuclear transfer (SCNT ES cells) are genetically identical to the donor, it will not generate rejection by the host's immune system and thus therapeutically may be more acceptable. Induced pluripotent stem cells (iPS) are a type of pluripotent stem cell artificially derived from an adult somatic cell by inducing a forced expression of a set of specific pluripotent genes. In the past few years, rapid progress in reprogramming and iPS technology has been made, and it seems to shadow any progress made in SCNT programs.

Objective: This review compares the application perspective of SCNT with that of iPS in regenerative medicine. Methods: We conducted a literature search using the MEDLINE (PubMed), Wiley InterScience, Springer, EBSCO, and Annual Reviews databases using the keywords "iPS”, "ES”, “SCNT” “induced pluripotent stem cells”, “embryonic stem cells”, "therapeutic cloning”, "regenerative medicine”, and "somatic cell nuclear transfer”. Only articles published in English were included in this review.

Results: These two methods both have advantages and disadvantages. Nevertheless, by using SCNT to generate patient-specific cell lines, it eliminates complications by avoiding the use of viral vectors during iPS generation. Success in in vitro matured eggs from aged women and even differentiation of oocytes from germ stem cells will further enhance the application of SCNT in regenerative medicine.

Conclusion: Human SCNT may be an appropriate mean of generating patient stem cell lines for clinical therapy in the near future.
\end{abstract}

Keywords: iPS, review, SCNT, therapeutic cloning

Abbreviations

iPS = Induced pluripotent stem cells

$\mathrm{ES}=$ Embryonic stem cells

SCNT $=$ Somatic cell nuclear transfer

Embryonic stem cells (ES) derived from embryos can be cultured indefinitely in large numbers and they also have the ability to differentiate into all 220 different cell types in our body and thus have great therapeutic potential. A number of cell types, including neural progenitors, dopaminergic (DA) neurons, insulinproducing cells, cardiomyocytes, endothelial cells, hematopoietic cells and many others, have been

Correspondence to: Kuldip Sidhu, Director, Stem Cell Lab Chair, Stem Cell Biology, Clinical Sciences Building, Prince of Wales Hospital, NSW 2052, Australia. E-mail: k.sidhu@unsw.edu.au generated from ES [1, 2]. Yet, destruction of embryos to isolate human embryonic stem cells (hES) is considered a major ethical concern that may limit the use of these cells for therapeutic purposes. Another limitation is the allogenic nature of these cells, and a risk of immune rejection when transplanted. Alternative techniques, somatic cell nuclear transfer (SCNT) and somatic cell reprogramming offer opportunities to overcome such transplantation issues such as graft rejection. However, SCNT technique raises other ethical issues as it requires the use of human eggs. Therapeutic cloning is the combination of SCNT and ES cell techniques to create specific ES cells that match those of a patient. Because ES cells derived by nuclear transfer (SCNT ES cells) are genetically identical to the donor, they will not generate rejection by the host's immune system and thus 
therapeutically may be more acceptable. Induced pluripotent stem cells (iPS) are a type of pluripotent stem cell artificially derived from an adult somatic cell by inducing a forced expression of a set of specific pluripotent genes. The past few years have witnessed the rapid progress in reprogramming and iPS technology, and this rapid progress appears to be overshadowing any progress made in SCNT programs. While reprogramming is still in its early stages and there are number of technical issues that need to be resolved before it can be used in clinical therapy, progress in SCNT on the other hand is at a slow pace, but nevertheless offering a number of clues in our understanding and optimizing the whole process of reprogramming. Here we have reviewed the progress made so far in both SCNT and reprogramming techniques and perspectives of these developments in regenerative medicine.

\section{SCNT in animals}

Previous studies have shown that SCNT ES-cells grow as immortal cell lines and produce pluripotent tumors when injected into immunocompromised mice [3]; germline transmission as indicated by tetraploid embryo complementation [4-6]; global gene-expression profiling of SCNT ES-cell lines derived from different donor-cell types revealed transcriptomes that are indistinguishable from those of fertilization-derived ES-cell lines [7]. Therefore, SCNT-cell lines derived by nuclear transfer are expected to have the similar therapeutic potential to those derived from fertilized embryos.

The differences between ES cell lines are simply associated with the different genetic background. Indeed, experiments in animals have shown that SCNT combined with ES cell represents a valid strategy for treating genetic disorders. SCNT embryos were constructed using nuclei from Parkinsonian mice and oocytes from healthy mice, and then 187 ES cell lines were generated from 24 Parkinsonian mice [8]. Cells were expanded and subjected to neural induction and differentiation into midbrain dopamine neurons. Each mouse received dopamine neuron progeny obtained from a SCNT-ES cell line derived from its own donor nuclei. Control mice received an allograft of dopamine neurons. Mice grafted with matched SCNT-cell-derived dopamine neurons showed a significant amelioration of the Parkinsonian phenotype in all behavioral tests, and superior graft survival and decreased immunogenicity compared with allogenic grafts [9]. These data further demonstrate the feasibility of treating individual Parkinsonian mice via therapeutic cloning.

An important problem raised at the present is the low efficiency of SCNT. For a donor nucleus to support development of an SCNT embryo, it must properly activate genes important for early embryonic development and suppress differentiation-associated genes that were transcribed in the original donor cell. Inadequate reprogramming of the donor nucleus is thought to be the principal reason for the developmental loss of most SCNT embryos. Comparative analysis of human-human, humanbovine, and human-rabbit cloned embryos at the morula stage has been conducted. It was found that appearances and the rates of development to the morula stage were similar, but the pattern of reprogramming of the donor genome was dramatically different. By contrast, with the interspecies clones, gene expression profiles of the human-human embryos showed extensive reprogramming of the donor nuclei, and that the expression pattern was similar in normal control embryos [10]. These results suggest that bovine and rabbit oocytes do not support appropriate embryonic genomic reprogramming of human somatic cell nuclei, and they were not able to generate patientspecific human stem cells.

In addition, inadequate reprogramming is also associated with the SCNT technique itself. The detrimental effect of fluorochrome bis-benzimide (Hoechst 33342) and ultraviolet (UV) light on the development and reprogramming of SCNT embryos has been identified in primates, because Hoechst 33342 and UV-light were able to damage to the oocyte through Hoechst 33342/UV-induced oocyte activation, and/or maturation promoting factor degradation, reaction of the residual Hoechst 33342 in cytoplasts with the introduced donor cell DNA [11, 12]. In rhesus macaque SCNT, an oocyte spindle imaging system was used to visualize the oocyte meiotic spindle during oocyte enucleation, avoiding the conventional use of Hoechst 33342 and UV light. A significant increase in the blastocyst formation rate (from $1 \%$ to $16 \%$ ) was achieved, and two embryonic stem cell lines were successfully isolated [11].

The differentiated state of the donor nucleus is critical for SCNT ES efficiency. Embryos cloned from embryonic stem cells faithfully express early embryonic genes [13], and cloning efficiency from ES or embryonic cells such as blastomeres (cells of the 
cleavage embryo) are roughly 10 to 20 times higher than that of cloning from somatic cells, possibly because these genes are already active in the donor genome. In addition, Blelloch et al. compared the cloning efficiencies using ES cells, cultured neural stem (NS) cells and differentiated fibroblasts as donor nuclei, respectively. Cloned blastocysts produced from NS cells gave rise to ES cells about as efficiently as did cloned blastocysts derived from ES cells, and about two to three times more efficiently than did cloned blastocysts generated from fibroblast donors. However, fibroblasts that had decreased levels of global DNA methylation were as efficient at being donors as were NS cells and ES cells, thus indicating that the epigenetic state of donor cells affects the reprogramming ability of cloned embryos [14]. To address this question further, a study was performed to create monoclonal mice using terminally differentiated lymphocytes. It was found that nuclei from mature $\mathrm{B}$ and $\mathrm{T}$ cells were able to direct development after being transferred into an oocyte, but this process was much less efficient than cloning using fibroblasts or cumulus cells [15].

The genome of adult stem cells that are present in the adult donor animal at low frequencies might resemble that of embryonic stem cells, because of their developmental immaturity, which is more amenable to or requires less reprogramming than the genome of a differentiated cell [4]. It will be interesting to produce patient specific SCNT embryos using purified adult stem cells from skin tissue, and of importance to the potential therapeutic application of autogenous cell nuclear transfer. However, the main problem encountered at present is how to isolate and purify adult stem cells, as well as identify specific markers.

Many studies found that SCNT embryos at various developmental stages, and even cloned offspring, show severe dysregulation of gene expression, with some genes being dysregulated in a donor-cell-dependent manner [16-18]. The persistence of donor-cell-specific gene expression in clones indicates the retention of an epigenetic memory of the donor nucleus [19]. Faithful reprogramming of the somatic genome and complete elimination of the epigenetic memory of the donor nucleus are required for successful cloning. Yet, by contrast, with fetal development, ES-cell derivation from cloned blastocysts is significantly more efficient than the potential of cloned blastocysts to develop into live offspring [11], because blastocyst formation and
ES-cell derivation are less restrained by genetic and epigenetic abnormalities.

\section{SCNT in humans}

Many studies have been conducted to produce human SCNT-ES using a regular somatic cell nuclear transfer technique [10, 20-22], but none have achieved the derivation of a stem cell line until recently [23]. French et al. [24] found, for the first time, that human oocytes can reprogram differentiated adult cells, and obtained $23 \%$ of blastocyst formation rate using two established adult male fibroblast lines and young oocytes. The development rate was similar to that observed in standard IVF blastocysts. Yet, a somatic cell nucleus was injected into a recipient oocyte without removing the oocyte genome, after artificial activation the resultant embryos containing two interphase nuclei were divided two groups by removing either the oocyte genome or somatic cell genome. Activated embryos containing only the somatic genome formed 4-12 cells, and then all arrested. By contrast, those embryos containing only the oocyte genome cleaved and 4/7 (57\%) developed to the blastocyst stage, and generated pluripotent parthenogenetic stem cells. Those oocytes containing two types of genomes were also able to develop to blastocysts, which also could generate pluripotent stem cells with tetraploid. It suggests that the presence of genetic material of the oocyte is required for development of SCNT embryos to the blastocyst stage [23]. The above data showed that the developmental competency of SCNT human embryos produced in different laboratories was different. To obtain human SCNT originated ES cells successfully; we should highlight the mechanism by which human oocytes support the reprogramming of differentiated somatic cells.

\section{Prospects of human SCNT vs. iPS technology}

At present, SCNT and iPS cell generation are most remarkable techniques used in reprogramming, and have received much interest in regenerative medicine. Both of them involve generating patientspecific stem cells containing the patient's DNA and genes. The primary purpose is to eliminate immune rejection between the patient and graft. At the same time, therapeutic cloning and iPS generation require an accessible source of somatic cells from a patient, usually dermal fibroblasts isolated from skin. Furthermore, these derived stem cell lines must also 
fulfil genetic matching with the initial donor skin cell. This is generally accomplished by performing microsatellite typing of short tandem repeat (STR) loci and single nucleotide polymorphisms (SNPs) [11]. However, for therapeutic applications, availability of human oocytes for reprogramming the somatic cell is limited by legal and social considerations.

Some SCNT studies have found that failure to generate SCNT-ES or unsuccessful reprogramming is the result of epigenetic abnormalities. Attempts have been made to alter epigenetic modifications; for example, histone acetylation [25] and DNA methylation [14], and the efficiency of generating SCNT-blastocysts and -ES lines are significantly increased. It is believed that successful generation of SCNT-ESs is possible. By using SCNT to generate patient-specific cell lines, it eliminates complications avoiding use of viral vectors during iPS generation. Human SCNT may be an appropriate mean of generating patient stem cell lines for clinical therapy. Generation of patient-specific cells via iPS technique involves transducing somatic cells with four critical pluripotent genes i.e. Oct4, Sox2, Klf4, c-Myc, or combinations thereof. To verify the pluripotent potential of iPS cells, their ability to differentiate into all lineages derived from three germ layers is essential. Conversion of iPS cells into cardiomyocytes [26], dopamine neurons [27], motor neurons [28], and pancreatic $\beta$ cells [29] have been reported. Recent research has focused on how to generate iPS cells without genomic integration through insertion of exogenous genes mediated by nonintegrating adenoviruses [30] or other [31] nongenetic modifying systems [32], including very recently by simply change in $\mathrm{pH}[33,34]$.

As the efficiency for generating iPS cells remains very low $(0.1 \%-1 \%)$, some chemicals can be used to increase reprogramming efficiency, such as DNMT inhibitor, AZA; HDAC inhibitor, TSA or VPA [35], and an inhibitor of histone methyltransferase G9a, BIX01294 was reported to not only increase efficiency, but also replacing Sox2 and c-Myc required in the reprogramming recipe [36].

However, there are still some differences between iPS cells and hES [37], because iPS cells are more variable in gene expression, DNA methylation and differentiation potentials [38]. More importantly, reprogramming to somatic cells seems to compromise genomic stability, introducing de novo mutations and copy number variations [39] in resultant iPS cells.
Derivation of stable iPS cell lines is critical for application of iPS, and will make an attractive potential tool in clinical therapy.

\section{Conclusion}

Reprogramming human somatic cells into iPS cells by expressing a pool of transcription factors seems far simpler and more efficient than generating ES cells using SCNT. It requires only conventional experimental equipment and small-scale laboratory work. The direct derivation of ES cells from SCNT requires special technical skill, a large number of oocytes and an array of special equipment.

Despite its simplicity, the reprogramming using transcription factors is relatively inefficient (less than $1 \%$ of treated fibroblasts were reprogrammed). In addition, in contrast to reprogramming by SCNT, the acquisition of pluripotency requires at least 3-4 weeks, without certainty of which sorts of cells can be reprogrammed. Not only does SCNT currently yield ES-like pluripotent cells, it also possesses a significant advantage because it does not use genomic alteration to introduce transcription factors. Moreover, mice derived from iPS cells generated by reprogramming factors appear more prone to cancer than their normal counterparts do, as it requires the forced expression of tumor-promoting factors [40].

Suppose iPS cells can be generated without genetic manipulation, and this has been achieved recently, this does not mean that SCNT can be discarded or is obsolete. Pressure from religion and ethics might shift the reprogramming method away from SCNT and toward iPS, SCNT allows the study of how epigenetic and genetic components contribute to the earliest steps of development, and it can explore mysteries of human life through SCNT embryos. By contrast, introducing transcription factors does not allow one to study embryo physiology, or the embryonic epigenome and transcriptome [41]. There are now more recent studies suggesting that in vitro matured eggs from aged women and even differentiation of oocytes from germ stem cells can be obtained [42], and, moreover, the success achieved by Noggle [43] will further enhance the application of SCNT in regenerative medicine.

The authors have no conflict of interest to declare.

\section{References}

1. Choumerianou DM, Dimitriou H, Kalmanti M. Stem 
cells: Promises versus limitations. Tissue engineering. Part B, Reviews. 2008; 14:53-60.

2. Sidhu KS, Ryan JP, Tuch BE. Derivation of a new human embryonic stem cell line, endeavour-1, and its clonal propagation. Stem Cells Dev. 2008: 17:41-51.

3. Munsie, MJ, Michalska AE, O’Brien CM, Trounson AO, Pera MF; Mountford PS. Isolation of pluripotent embryonic stem cells from reprogrammed adult mouse somatic cell nuclei. Curr Biol. 2000; 10:989-92.

4. Hochedlinger K, Jaenisch R. Nuclear transplantation, embryonic stem cells, and the potential for cell therapy. N Engl J Med. 2003; 349:275-96.

5. Eggan K, Baldwin K, Tackett M, Osborne J, Gogos J, Chess A, et al. Mice cloned from olfactory sensory neurons. Nature 2004; 428:44-9.

6. Li J, Ishii T, Feinstein P, Mombaerts P. Odorant receptor gene choice is reset by nuclear transfer from mouse olfactory sensory neurons. Nature. 2004; 428:393-9.

7. Brambrink T, Hochedlinger K, Bell G, Jaenisch R. ES cells derived from cloned and fertilized blastocysts are transcriptionally and functionally indistinguishable. PNAS. 2006; 103:933-8.

8. Al-Shamy G, Ohta H, Wakayama T, Studer L. Therapeutic cloning in individual parkinsonian mice. Nature. 2008; 14:379-81.

9. Tabar V, Tomishima M, Panagiotakos G, Wakayama S, Menon J, Chan B, et al. Differentiation of embryonic stem cell lines generated from adult somatic cells by nuclear transfer. Science. 2001; 292:740-3.

10. Chung Y, Bishop CE, Treff NR, Walker SJ, Sandler VM, Becker S, et al. Reprogramming of human somatic cells using human and animal oocytes. Cloning Stem Cells. 2009; 11:213-23.

11. Byrne JA, Pedersen DA, Clepper LL, Nelson M, Sanger WG, Gokhale S, et al. Producing primate embryonic stem cells by somatic cell nuclear transfer. Nature. 2007; 450:497-502.

12. Gurdon J. Primate therapeutic cloning in practice. Nature. 2008; 26:64-5.

13. Bortvin A, Eggan K, Skaletsky H, Akutsu H, Berry DL, Yanagimachi R, et al. Incomplete reactivation of Oct4-related genes in mouse embryos cloned from somatic nuclei. Development. 2003; 130:1673-80.

14. Blelloch R, Wang Z, Meissner A, Pollard S, Smith A, Jaenisch R. Reprogramming efficiency following somatic cell nuclear transfer is influenced by the differentiation and methylation state of the donor nucleus. Stem Cells. 2006; 24:2007-13.

15. Wakayama T, Yanagimachi R. Mouse cloning with nucleus donor cells of different age and type. Mol
Reprod Dev. 2001; 58:376-83.

16. Humpherys D, Eggan K, Akutsu H, Friedman A, Hochedlinger K, Yanagimachi R, et al. Abnormal gene expression in cloned mice derived from embryonic stem cell and cumulus cell nuclei. Proc Natl Acad Sci USA. 2002; 99:12889-94.

17. Ng RK, JB Gurdon. Epigenetic memory of active gene transcription is inherited through somatic cell nuclear transfer. Proc Natl Acad Sci USA. 2005; 102:1957-62.

18. Kohda T, Inoue K, Ogonuki N, Miki H, Naruse M, Kaneko-Ishino T, et al. Variation in gene expression and aberrantly regulated chromosome regions in cloned mice. Biol Reprod. 2005; 73:1302-11.

19. Jaenisch R. Human cloning-the science and ethics of nuclear transplantation. N Engl J Med. 2004; 351: 2787-91.

20. Hall VJ, Compton D, Stojkovic P, Nesbitt M, Herbert M, Murdoch A, et al. Developmental competence of human in vitro aged oocytes as host cells for nuclear transfer. Hum Reprod. 2007; 22:52-62.

21. Egli D, Chen AE, Saphier G, Powers D, Alper M, Katz $\underline{K}$, et al. Impracticality of egg donor recruitment in the absence of compensation. Stem Cell. 2011; 9: 293-4.

22. Stojkovic M, Stojkovic P, Leary C, Hall VJ, Armstrong $\mathrm{L}$, Herbert M, et al. Derivation of a human blastocyst after heterologous nuclear transfer to donated oocytes. Reprod Biomed Online. 2005; 11:226-31.

23. Tachibana M, Amato P, Sparman M, Gutierrez NM, Tippner-Hedges R, Ma H, et al. Human embryonic stem cells derived by somatic cell nuclear transfer. Cell. 2013;153:1228-38.

24. French AJ, Adams CA, Anderson LS, Kitchen JR, Hughes MR, SH Wood. Development of human cloned blastocysts following somatic cell nuclear transfer with adult fibroblasts. Stem cells. 2008; 26:485-93.

25. Zhao J, Ross JW, Hao Y, Spate LD, Walters EM, Samuel MS, et al. Significant improvement in cloning efficiency of an inbred miniature pig by histone deacetylase inhibitor treatment after somatic cell nuclear transfer. Biol Reprod. 2009; 81:525-30.

26. Zhang J, Wilson GF, Soerens AG, Koonce CH, Yu JY, Palecek SP, et al. Functional cardiomyocytes derived from human induced pluripotent stem cells. Circ Res. 2009; 104:30-41.

27. Cai J, Yang M, Poremsky E, Kidd S, Schneider JS, Iacovitti L. Dopaminergic neurons derived from human induced pluripotent stem cells survive and integrate into 6-OHDA lesioned rats. Stem Cells Dev. 2010; 19: 1017-23. 
28. Karumbayaram S, Novitch BG, Patterson M, Umbach JA, Richter L, Lindgren A, et al. Directed differentiation of human-induced pluripotent stem cells generates active motor neurons. Stem Cells. 2009; 27:806-11.

29. Zhang J, Wilson GF, Soerens AG, Koonce CH, Yu JY, Palecek SP, et al. Functional cardiomyocytes derived from human induced pluripotent stem cells. Circ Res. 2009; 104:30-41.

30. Stadtfeld M, Nagaya M, Utikal J, Weir G, Hochedlinger K. Induced pluripotent stem cells generated without viral integration. Science. 2008; 322:945-9.

31. Yu J, Hu K, Smuga-Otto K, Tian SL, Stewart R, Slukvin II, et al. Human induced pluripotent stem cells free of vector and transgene sequences. Science. 2009; 324:1266.

32. Zhou H, Wu S, Joo JY, Zhu S, Han DW, Lin T, et al. Generation of induced pluripotent stem cells using recombinant proteins. Cell Stem Cell. 2009; 4:381-4.

33. Obokata H, Wakayama T, Sasai Y, Kojima K, Vacanti MP, Niwa H, et al. Stimulus-triggered fate conversion of somatic cells into pluripotency. Nature. 2014; 505: 641-58.

34. Obokata H, Sasai Y, Niwa H, Kadota M, Andrabi M, Takata N, et al. Bidirectional developmental potential in reprogrammed cells with acquired pluripotency. Nature Lett. 2014; 505:676-86.

35. Huangfu D, Maehr R, Guo WJ, Eijkelenboom A, Snitow M, Chen AE, et al. Induction of pluripotent stem cells by defined factors is greatly improved by small-molecule compounds. Nat Biotech. 2008; 26:
795-7.

36. Shi Y, Do JT, Desponts C, Hahm HS, Scholer HR, Ding S. A combined chemical and genetic approach for the generation of induced pluripotent stem cells. Cell Stem Cell. 2008; 3:119.

37. Wilson KD, Venkatasubrahmanyam S, Jia F, Sun N, Butte AJ, JC Wu. MicroRNA profiling of humaninduced pluripotent stem cells. Stem Cells Dev. 2009; 18:749-58.

38. Hu BY, Weick JP, Yu J, Ma LX, Zhang XQ, Thomson JA, et al. Neural differentiation of human induced pluripotent stem cells follows developmental principles but with variable potency. Proc Natl Acad Sci USA. 2010; 107:4335-40.

39. Mayshar Y, Ben-David U, Lavon N, Biancotti JC, Yakir B, Clark AT, et al. Identification and classification of chromosomal aberrations in human induced pluripotent stem cells. Cell Stem Cell. 2010; 7:521-31.

40. Marion RM, Strati K, Li H, Murga M, Blanco R, Ortega S, et al. Ap53-mediated DNA damage response limits reprogramming to ensure iPS cell genomic integrity. Nature. 2009; 460:1149-53.

41. Zwaka TP. What comes after iPS? Nature Reports Stem Cells. 2008, doi:10.1038/stemcells.2008.54.

42. White YAR, Woods DC, Takai Y, Ishihara O, Seki H, JL Tilly. Oocyte formation by mitotically active germ cells purified from ovaries of reproductive-age women. Nature. 2012; 18:413-22.

43. Noggle S, Fung HL, Gore A, Martinez H, Satriani KC, Prosser R, et al. Human oocytes reprogram somatic cells to a pluripotent state. Nature. 2011; 478:70-7. 